\title{
Relaciones entre la investigación y la acción en didáctica de las matemáticas
}

\author{
Josep Gascón, Universitat Autònoma de Barcelona (España) \\ Pedro Nicolás, Universidad de Murcia (España)
}

\section{Relaciones entre la investigación y la acción en didáctica de las matemáticas \\ Resumen}

En trabajos previos iniciamos una discusión, con diferentes investigadores en didáctica de las matemáticas, sobre el presunto carácter normativo o prescriptivo de la ciencia didáctica. Esta discusión derivó, en una segunda etapa, hacia la necesidad de explicitar los postulados o asunciones básicas y los fines (educativos y de la investigación) que cada teoría en didáctica de las matemáticas propugna o asume. Con el presente trabajo, que inaugura una tercera etapa del diálogo, queremos abordar el difícil problema de las relaciones entre la investigación y la acción en didáctica de las matemáticas. Para afrontar este problema desde la perspectiva de la teoría antropológica de lo didáctico describimos y ejemplificamos dos tipos particulares de resultados de la investigación didáctica muy relacionados entre sí, y mostramos para qué y cómo pueden utilizarse dichos resultados con el objetivo de incidir, simultáneamente, sobre la investigación y la acción didácticas.

Palabras clave. Diálogo entre teorías didácticas; paradigmas didácticos; resultados de la investigación; leyes didácticas; economía y ecología del paradigma didáctico vigente.

\section{Relationship between teaching and research in didactics of mathematics}

\section{Abstract}

In previous works we started a dialogue, with different researchers in didactics of mathematics, about the presumed normative or prescriptive character of didactics as a scientific activity. This dialogue led to a second step in which all the scholars involved tried to make explicit the postulates or basic assumptions of their own approach in didactics, as well as the (research and educational) ends assumed by that approach. With the present work, placed in a third step of the dialogue, we want to face the difficult question of the relationship between research in didactics of mathematics and teaching mathematics. To address this problem, we describe and illustrate, from the perspective of the anthropological theory of the didactics, two particular kinds of closely related research results. Then we show how, and to what end, those results can be used in order to influence both research and teaching simultaneously.

\section{Key words}

Dialogue between theories in didactics; didactic paradigm; research result; didactic law; economy and ecology of the current didactic paradigm.

\section{Contexto del diálogo entre teorías didácticas}

Este trabajo se sitúa en el contexto de un diálogo entre teorías didácticas, cuyos orígenes se remontan al año 2013, con la pregunta que planteamos a Guy Brousseau sobre el presunto carácter normativo o prescriptivo de la ciencia didáctica. Luego 
extendimos la pregunta a un amplio conjunto de colegas ${ }^{1}$. Sus respuestas fueron publicadas en la lengua original y en inglés en Gascón y Nicolás (2017).

El diálogo se desarrolló incluyendo nuevas cuestiones relativas a los postulados o asunciones básicas de cada teoría, a los fines que persigue la investigación didáctica y a los resultados que cada teoría considera admisibles. En torno a estas cuestiones tuvo lugar una segunda etapa del diálogo plasmada en múltiples trabajos publicados ${ }^{2}$. Esta etapa culminó en un curso avanzado presencial organizado por el Centre de Recerca Matemàtica (Barcelona) con ocasión del Intensive Research Program entre el 3 y 14 de junio de 2019 y cuyos trabajos están en vías de publicación (Chevallard et al., en prensa).

Quedaron muchas cuestiones abiertas relativas a las complejas relaciones entre investigación didáctica y práctica educativa. Con el objetivo de abordar estas cuestiones, hemos convocado en Gascón y Nicolás (2021) la tercera etapa del diálogo. Como argumentamos en dicho trabajo, para profundizar en el diálogo entre teorías didácticas y, en particular, para comparar las diferentes posturas en lo que hace referencia a las relaciones entre la investigación y la acción didácticas, será preciso contrastar los fines educativos (relativos a la educación matemática) y los modelos epistemológicos (de las matemáticas) asumidos por cada una de ellas. Aparece aquí una idea que suele pasar inadvertida y que desempeñará un papel central en nuestra aportación a esta etapa del diálogo. Sintéticamente, puede enunciarse como sigue: toda investigación didáctica tiende a asumir, de facto, de manera más o menos explícita, una forma particular de conceptualizar los conocimientos matemáticos (un modelo epistemológico disciplinar junto a modelos epistemológicos específicos, subdisciplinares, de los diferentes ámbitos de las matemáticas) y, en coherencia con dichos modelos, unos fines de la educación matemática, esto es, una repuesta al para qué estudiar matemáticas (o un dominio particular de las mismas) en una institución determinada.

En coherencia con esta convicción y con el objetivo de lanzar la discusión, hemos propuesto a diferentes investigadores en didáctica de las matemáticas un conjunto de cuestiones en las que intervienen, además de los fines educativos y el modelo epistemológico, la forma como se utiliza en la bibliografía didáctica la noción, muy controvertida, de "mejorar la enseñanza de las matemáticas".

¿Qué se entiende por "aplicación o implementación de los resultados de la investigación en didáctica de las matemáticas"? ¿Qué tipo de "resultados de la investigación" se supone que pueden aplicarse a la acción didáctica y cómo puede llevarse a cabo dicha aplicación? ¿Qué papel desempeñan el modelo epistemológico y los fines de la educación matemática que se asumen en la formulación de los resultados de la investigación? ¿Qué se entiende por "mejorar" los procesos de estudio o enseñanza-aprendizaje de las matemáticas?

En lo que sigue, proponemos nuestra respuesta (provisional) a algunas de estas cuestiones desde la perspectiva de la teoría antropológica de lo didáctico (TAD).

Dado que toda teoría didáctica, más allá de un modelo epistemológico y unos fines de la educación matemática, tiende a asumir en la práctica ciertos paradigmas didácticos

\footnotetext{
${ }^{1}$ En esta etapa participaron: Guy Brousseau (TSD), Michèle Artigue (Contraste entre diversas teorías), Ed Dubinsky (APOS), María Trigueros (APOS), Juan D. Godino (EOS), Koeno Gravemeijer (Educación Matemática Realista), Ricardo Cantoral (TSME), y Josep Gascón y Pedro Nicolás (TAD).

2 Lerman (2018), Proulx (2018), Bartolini Bussi (2018), Davis (2018), Oktaç et al. (2019), Godino et al. (2019), Staats y Laster (2019), y Gascón y Nicolás (2019b, 2019c).
} 
como propios y que estos paradigmas condicionan simultáneamente la manera como se interpretan los resultados de la investigación y como se utilizan en la acción didáctica, empezamos introduciendo la noción de paradigma didáctico.

\section{Paradigmas didácticos}

Un paradigma didáctico disciplinar, en el caso de las matemáticas, relativo a una institución escolar I, constituye una forma particular de interpretar la educación matemática en I. Está caracterizado esencialmente por un modelo epistemológico (ME) de las matemáticas (actual o potencialmente vigente en I) y por los fines educativos (F) relativos a la educación matemática, que dicho paradigma propugna. Estos fines se formulan con los términos y las nociones que proporciona el ME. Un análisis minucioso del ME permite prever con bastante precisión el tipo de fines que se pueden propugnar $\mathrm{y}$, recíprocamente, los fines que se propugnan para la educación matemática en una institución determinada desvelan algunas características importantes del ME subyacente y permiten vislumbrar los fenómenos didácticos que serán visibles en dicha institución. Así, por ejemplo, si entre los fines educativos se encuentra el aprendizaje de conocimiento proposicional, los argumentos desempeñarán un papel esencial en las praxeologías constitutivas del modelo epistemológico (Gascón y Nicolás, en prensa).

Junto con el ME y los $\mathrm{F}$, en un paradigma didáctico concurren los medios didácticos (MD) que se suponen adecuados para alcanzar los fines y los fenómenos didácticos $(\varphi)$ a los que el paradigma responde. Esto es, un paradigma didáctico (PD) no surge de la nada, sino como reacción a hechos didácticos que se interpretan como indicadores de los citados fenómenos (que el nuevo paradigma pretende prevenir o favorecer). En resumen, un paradigma didáctico se caracteriza por cuatro componentes concurrentes:

$$
\mathrm{PD}=[\mathrm{ME}, \mathrm{F}, \mathrm{MD}, \varphi]
$$

Esta noción de "paradigma didáctico" presenta una cierta unidad funcional, por lo que sus componentes deben analizarse conjuntamente, como sistema complejo. Los F que un PD propugna están condicionados por el ME en el que se sustenta dicho paradigma. Conjuntamente, el ME y los F, influyen, a su vez, sobre el tipo de prácticas docentes que los medios didácticos MD permiten llevar a cabo (Gascón, 2001) y, en particular, condicionan el tipo de conocimiento que aspira a trasmitir la institución en cuestión. Asimismo, el ME y los F delimitan los hechos didácticos visibles en los correspondientes procesos de estudio, así como su interpretación como fenómenos.

Dada una institución escolar I, y un determinado periodo histórico, distinguimos entre el paradigma didáctico vigente en $\mathrm{I}\left(\mathrm{PDV}_{\mathrm{I}}\right)$ a lo largo de dicho periodo, y los paradigmas didácticos de referencia $\left(\mathrm{PDR}_{\mathrm{I}}\right)$ que construye una teoría didáctica - como, por ejemplo, la TAD — como instrumentos útiles para analizar el $\mathrm{PDV}_{\mathrm{I}} \mathrm{o}$ los paradigmas didácticos posibles en I. Es muy importante subrayar que un PDR es un tipo ideal en el sentido de Weber (1904/2009), esto es, un modelo teórico, un caso límite que no ha existido nunca ni puede existir en estado puro en ninguna institución. Es precisamente la comparación entre el tipo ideal de paradigma y la realidad empírica (la contingencia histórica), la que proporciona la posibilidad de formular hipótesis explicativas de los hechos empíricos y de su relación con otros fenómenos. 
Si $\mathscr{D}$ representa un cierto dominio de las matemáticas que viven en una institución I (y que, eventualmente, puede abarcar el conjunto de las matemáticas globalmente consideradas), entonces PDV $_{\mathrm{I}}(\mathscr{D})$ constituye una representación ${ }^{3}$ de la manera, más o menos explícita, como los sujetos de I interpretan la educación matemática en torno a $\mathscr{D}$, esto es, de la forma como describen y relacionan entre sí: el modelo epistemológico vigente en I en torno a $\mathscr{D}, \operatorname{MEV}_{\mathrm{I}}(\mathscr{D})$; los fines educativos, $\mathrm{F}_{\mathrm{V}}(\mathfrak{D})$, que se persiguen en $\mathrm{I}$ con el estudio de $\mathscr{D}$; los medios, $\operatorname{MDV}_{\mathrm{I}}(\mathscr{D})$, que se utilizan en I para alcanzar dichos fines; y los fenómenos didácticos, $\varphi \mathrm{v}(\mathscr{D})$, que el $\operatorname{MEV}_{\mathrm{I}}(\mathscr{D})$ saca a la luz y a los que el $\operatorname{PDV}_{I}(\mathscr{D})$, globalmente considerado, responde.

$$
\operatorname{PDV}_{\mathrm{I}}(\mathscr{D})=\left[\mathrm{MEV}, \mathrm{F}_{\mathrm{V}}, \mathrm{MDV}, \varphi_{\mathrm{V}}\right]
$$

Resulta, en definitiva, que el $\operatorname{PDV}_{\mathrm{I}}(\mathcal{D})$ caracteriza, y en cierto sentido rige, aunque sea implícitamente, la estructura y el funcionamiento del estudio de $\mathscr{D}$ en $\mathrm{I}^{4}$.

Análogamente, caracterizamos los PDR que se construyen desde la investigación didáctica para analizar los PDV en una institución o los paradigmas didácticos posibles, mediante cuatro componentes:

$$
\operatorname{PDR}_{\mathrm{I}}(\mathscr{D})=\left[\mathrm{MER}, \mathrm{F}_{\mathrm{R}}, \mathrm{MDR}, \varphi_{\mathrm{R}}\right]
$$

El modelo epistemológico de referencia, $\operatorname{MER}_{\mathrm{I}}(\mathscr{D})$, es una forma de redefinir o reinterpretar el dominio $\mathscr{D}$, alternativa a la descripción oficial basada en el $\operatorname{MEV}_{\mathrm{I}}(\mathfrak{D})$; $\mathrm{F}_{\mathrm{R}}(\mathcal{D})$ se refiere a los fines educativos de referencia que dicho paradigma propugna; $\operatorname{MDR}_{\mathrm{I}}(\mathscr{D})$ designa los medios considerados necesarios para lograr, mediante el estudio de $\mathscr{D}$, los fines $\mathrm{F}_{\mathrm{R}}(\mathfrak{D})$; y $\varphi_{\mathrm{R}}(\mathscr{D})$ son los fenómenos didácticos a los que el $\operatorname{PDR}_{\mathrm{I}}(\mathscr{D})$ pretende responder. Son fenómenos invisibles desde la perspectiva que proporciona el PDV y sólo se revelan completamente cuando analizamos el PDV a la luz de cierto PDR.

En resumen, podemos considerar que las nociones de PDR y PDV generalizan respectivamente y completan las antiguas nociones de MER y de MEV. En particular, un PDR I(D) se construye como alternativa al paradigma vigente, $\operatorname{PDV}_{\mathrm{I}}(\mathscr{D})$, de la misma forma que la construcción de un MER constituía una alternativa al MEV en una institución y un instrumento para estudiarlo. Veremos, en lo que sigue, que tanto la formulación de un tipo particular de resultados de la investigación didáctica como su posible utilización para actuar en el universo didáctico, dependerá del juego entre el PDV y los PDR que se utilicen para estudiarlo.

\section{Resultados que la didáctica puede formular legítimamente}

La noción de "resultado de la investigación didáctica" se utiliza, de manera más o menos explícita, en los discursos didácticos, pero muy raramente se toma como objeto de estudio en sí misma. No suele plantearse qué es un resultado de la investigación di-

\footnotetext{
${ }^{3}$ El análisis y la explicitación de esta representación se elabora desde la perspectiva de cierto PDR.

${ }^{4}$ En este trabajo las nociones de institución y de dominio de las matemáticas que vive en una institución son consideradas como nociones primitivas. En cuanto al estudio (de un dominio en una institución), hay que recordar que se utiliza, en la TAD, como una noción muy general. Incluye la génesis, la enseñanza, el aprendizaje, la difusión y la aplicación de los conocimientos de todo tipo vinculados de algún modo a dicho dominio. Abreviadamente podemos decir que, en este sentido, la didáctica es la ciencia del estudio, incluyendo la ayuda al estudio (Gascón, 1997).
} 
dáctica ni, mucho menos, qué tipo de resultados puede la didáctica formular legítimamente. Desde la perspectiva de la TAD, nos hemos planteado esta cuestión y hemos empezado a responderla en Gascón y Nicolás (2019c) y Gascón (2021).

Empezaremos por caracterizar un tipo de cuestiones didácticas cuyas respuestas podrán ser consideradas como resultados de la investigación didáctica. Según la forma como la TAD conceptualiza la actividad humana, los objetos y procesos que pueblan el universo didáctico tienen estructura praxeológica. En consecuencia, en una primera aproximación, podríamos decir que la didáctica estudia, entre otras cosas, las condiciones y restricciones que inciden en la vida de las praxeologías relativas al estudio ${ }^{5}$ en las instituciones de la sociedad o, en otros términos, la economía y la ecología de dichas praxeologías. Estos son ejemplos de tipos de cuestiones que forman parte de la problemática económico-ecológica de las praxeologías relativas al estudio:

- ¿Cuál es la estructura y la dinámica (la economía) de las praxeologías que viven en una institución determinada (entre las que se incluyen los procesos didácticos y los productos del estudio)?

- ¿Por qué las praxeologías han llegado a ser como son en dicha institución?

- ¿Qué condiciones se requieren para modificar las praxeologías en una dirección determinada?

- ¿Qué restricciones dificultan o impiden esta modificación?, ¿qué consecuencias acarrearía?

Pero la economía y ecología de las praxeologías matemáticas y didácticas que viven en una institución escolar (e. g. la estructura y la dinámica de las praxeologías o las condiciones que se requieren para modificarlas en una dirección determinada) están fuertemente condicionadas por la codeterminación entre ambos tipos de praxeologías, por los fines educativos que se persiguen y, en última instancia, por el PDV en dicha institución. Por tanto, el estudio de la economía y la ecología deberá trasladarse desde las praxeologías, consideradas aisladamente, a los PDV en la institución en cuestión, como sistemas complejos ${ }^{6}$. Se obtiene, de esta forma, una ampliación de la problemática económico-ecológica que precisamos en lo que sigue.

En todos los casos, para responder a las cuestiones relativas a la economía y la ecología del PDV $(\mathscr{D})$, se utiliza necesariamente un punto de vista, un sistema de referencia proporcionado por un $\operatorname{PDR}_{\mathrm{I}}(\mathscr{D})^{7}$. Recordemos que un $\operatorname{PDR}_{\mathrm{I}}(\mathscr{D})$ es una hipótesis científica, construida desde la investigación, que constituye una forma de interpretar la educación matemática en $\mathrm{I}$, en torno al dominio $\mathscr{D}$, alternativa a la que encarna el $\operatorname{PDV}_{\mathrm{I}}(\mathscr{D})$. En consecuencia, tomando como referencia un $\operatorname{PDR}_{\mathrm{I}}(\mathscr{D})$, construido como alternativa al

${ }^{5}$ Son las praxeologías que describen las actividades encaminadas a la ampliación del conocimiento.

${ }^{6}$ Un sistema complejo presenta un comportamiento global y características de conjunto que no pueden reducirse al comportamiento y propiedades de sus componentes aisladamente. La complejidad de un sistema no está solamente determinada por la heterogeneidad de los elementos (o subsistemas) que lo componen. La característica determinante de un sistema complejo es la interdefinibilidad y mutua dependencia de las funciones que cumplen dichos elementos dentro del sistema total (García, 2011).

${ }^{7}$ En la investigación didáctica la construcción de un $\operatorname{PDR}_{I}(\mathscr{D})$ se realiza en paralelo, simultáneamente, a la del $\operatorname{PDV}_{\mathrm{I}}(\mathscr{D})$ que se pretende analizar. 
$\operatorname{PDV}_{\mathrm{I}}(\mathfrak{D})$, podemos plantear las siguientes cuestiones que forman parte de la problemática económico-ecológica del $\operatorname{PDV}_{\mathrm{I}}(\mathscr{D})$ :

- ¿Cuál es la estructura y la dinámica (la economía) del $\operatorname{PDV}_{\mathrm{I}}(\mathscr{D})$ considerado como un sistema complejo? ¿Por qué el $\operatorname{PDV}_{\mathrm{I}}(\mathscr{D})$ ha llegado a ser como es en dicha institución?

- ¿Qué fenómenos didácticos, invisibles desde la perspectiva del $\operatorname{PDV}_{\mathrm{I}}(\mathscr{D})$, saca a la luz y permite explicar el $\operatorname{PDR}_{\mathrm{I}}(\mathfrak{D})$ ?

- ¿Qué condiciones se requieren para modificar el $\operatorname{PDV}_{\mathrm{I}}(\mathscr{D})$ en la dirección que marca el PDR $_{\mathrm{I}}(\mathscr{D})$ considerado? En particular, ¿qué condiciones son necesarias para modificar el $\operatorname{MEV}_{\mathrm{I}}(\mathscr{D})$ en la dirección del $\operatorname{MER}_{\mathrm{I}}(\mathfrak{D})$ y los $\mathrm{F}_{\mathrm{V}}$ en la dirección de los $\mathrm{F}_{\mathrm{R}}$ ? \&Y para modificar los $\operatorname{MDV}_{\mathrm{I}}(\mathscr{D})$ en la dirección de los $\mathrm{MDR}_{\mathrm{I}}(\mathscr{D})$ ?

- ¿Qué restricciones dificultan la realización de dichas modificaciones y qué consecuencias acarrearían?

- ¿Qué elementos de los fines de referencia $F_{R}$ justifican el estudio del dominio $\mathscr{D}$ en la institución I y cómo se relacionan con los fines educativos vigentes $\mathrm{F}_{\mathrm{V}}$ ?

Como se explica en Gascón y Nicolás (2017, 2019b, 2019c) y en Gascón (2021), las siguientes cuestiones pertenecen a la "esfera de los valores" (Weber, 1917/2010), de modo que las posibles respuestas no pueden considerarse resultados de la investigación científica:

- ¿Qué debemos hacer si el PDV $\operatorname{PD}_{\mathrm{I}}(\mathcal{D})$ da origen a procesos de estudio con características incompatibles con los postulados que se asumen desde la investigación?

- ¿Cómo valoramos este tipo de $\operatorname{PDV}_{\mathrm{I}}(\mathfrak{D})$ y los procesos de estudio que determina?

- ¿Debemos hacer algo para que el $\operatorname{PDV}_{\mathrm{I}}(\mathfrak{D})$ se modifique en una dirección determinada y, en este caso, en qué dirección?

- Entre los diferentes tipos posibles de $\operatorname{PDR}_{\mathrm{I}}(\mathscr{D})$ “ideales”, ¿cuál de ellos es mejor?

Después de formular algunos tipos de cuestiones didácticas, indicamos el tipo de resultados que la ciencia didáctica puede enunciar legítimamente como respuesta a dichas cuestiones. En la TAD, los resultados de la investigación se formulan mediante leyes didácticas, que responden a las cuestiones de la problemática didáctica, y mediante explicaciones de fenómenos didácticos:

La TAD prend position, [...] contre l'oubli des phénomènes et des lois didactiques, qui tend à réduire la didactique à l'étude de l'empirie sans médiation théorique. (Chevallard, 2011, p. 24)

El contraste entre el $\operatorname{PDV}_{\mathrm{I}}(\mathscr{D})$ — que rige la educación matemática en torno al dominio $\mathscr{D}$ en I- y la interpretación que propone cierto $\operatorname{PDR}_{\mathrm{I}}(\mathscr{D})$ como alternativa hipotética, permite formular leyes didácticas que describen y explican determinados fenómenos didácticos. Sin pretender dar una definición de "fenómeno didáctico" (como tampoco tenemos una definición de "fenómeno físico, biológico, económico, psicológico o social") diremos brevemente lo que entendemos por "fenómeno (científico)":

Un fenómeno es un hecho notable que admite una descripción genérica y se repite regularmente en determinadas circunstancias. Inicialmente asombroso, el fenómeno demanda una 
explicación científica; luego, cuando ya la tiene, permite corroborar las leyes y teorías invocadas para explicarlo. (Mosterín y Torretti, 2010, p. 243)

Una "explicación (científica)" es una respuesta (que cumple determinadas condiciones) a una pregunta del tipo:

“¿Por qué ocurre el suceso e (en vez de los sucesos $\left.d_{1}, d_{2}, \ldots\right)$ ?”

En ella tenemos, junto a lo que requiere una explicación (explanandum), lo que proporciona la explicación (explanans), y la relación explicativa entre el explanans y el explanandum. Normalmente el explanans contiene un enunciado general que es una ley. Volveremos sobre esta cuestión formulando preguntas concretas cuya respuesta constituiría una explicación de ciertos fenómenos didácticos (ver final del apartado 4).

En cuanto a lo que se entiende por "ley científica", al igual que sucede con la noción de "explicación científica", esta es una cuestión muy controvertida en filosofía de la ciencia. Simplificando mucho, podemos decir que su estructura lógica es la siguiente:

\section{"Todos los sucesos del tipo A son también del tipo B"}

En el caso de las leyes que la didáctica puede formular legítimamente, nuestra respuesta a la cuestión de la normatividad fue inequívoca: la ciencia (en particular, la didáctica) no está legitimada para enunciar, como resultados de la investigación, ni juicios de valor, ni prescripciones normativas de ningún tipo relativas a cómo deben diseñarse y gestionarse los procesos de estudio. Esta tesis niega la legitimidad de la ciencia didáctica para valorar unos fines educativos por encima de otros y, en definitiva, para "preferir" o considerar que un paradigma didáctico es "mejor" o "más valioso" que otro (Gascón y Nicolás, 2016/2019a, 2017, 2019c, 2021). Lo que no niega esta tesis es la incidencia de valores de todo tipo en todos los aspectos de la actividad humana y, en particular, en la actividad científica, tal como señalamos en la primera etapa del diálogo:

"Every human activity taking place in society (in particular, scientific activity) is conditioned by several systems of values and regulated by more or less explicit norms. Concerning scientific activity, one could distinguish between epistemic and non-epistemic values. Epistemic values are linked to the standards of scientific truth: objectivity, internal coherence, compatibility with other accepted theories, predictive power, etc. Among those nonepistemic values affecting scientific activity might be social utility, social cost, accessibility, etc. Epistemic and non-epistemic values, and the corresponding rules derived from them, affect scientific activity as a whole. They have an effect at different moments of this activity, including the choice of research problems, the selection of empirical data and their treatment, the formulation of scientific results and their interpretation, dissemination, and use" (Gascón y Nicolás, 2017, p. 9).

El hecho de que en la actividad científica sea imprescindible utilizar juicios de valor para tomar decisiones de todo tipo, no significa que dichas decisiones puedan establecerse racionalmente como resultados de la investigación científica. Así, para que tenga sentido la expresión "mejorar los procesos de estudio o los procesos de enseñanzaaprendizaje de las matemáticas" es imprescindible asumir un paradigma didáctico y, en particular, unos fines educativos específicos que, como todos, son postulados que no pueden establecerse racionalmente. En consecuencia, todas las decisiones que se tomen para "mejorar los procesos de estudio" dependerán de dichos postulados.

Entre las decisiones más radicales - que siempre comportan asunciones- que se toman en la actividad científica están las de elegir en qué marco teórico se trabajará, qué objetos y qué relaciones poblarán el universo (su ontología), qué constructos teóricos se utilizarán y qué postulados se asumirán provisionalmente. En el caso de la investigación 
didáctica, se debe decidir, entre otras cosas, el modelo epistemológico de las matemáticas y los fines educativos que se asumen (aunque sea provisionalmente). No parece que los problemas que plantean estas decisiones puedan ser zanjados en ningún caso, apelando a resultados de la investigación científica.

Para intentar solventar estos problemas, algunos enfoques apelan a los "juicios de valor compartidos por la comunidad didáctica". Pero, en el caso de las ciencias sociales (aunque no sólo en ellas), hablar de "juicios de valor compartidos por una comunidad" es muy problemático. En el caso de la didáctica, es posible constatar una rápida evolución de la ideología dominante en la comunidad educativa (incluyendo la comunidad didáctica) fuertemente condicionada por ciertos valores no epistémicos.

According to Bourdieu (2001), social sciences are strongly exposed to non-epistemic values because their object of study is too important, from the point of view of social and symbolic order, to allow for autonomous development. Some of the features described by Bourdieu for the whole family of social sciences are particularly present in the case of didactics. For instance, the influence of heteronomous verdicts is very strong, especially the demand of social usefulness and the obedience to the dictates of the predominant pedagogical ideology. (Gascón y Nicolás, 2017, p. 9)

Si la comunidad didáctica llegara a compartir determinados "juicios de valor", lo cual es muy improbable, significaría que se ha producido cierta confluencia entre los postulados o asunciones básicas de las diferentes teorías. Incluso así, los juicios de valor seguirían sustentándose en los citados postulados (más o menos compartidos) que nunca pueden establecerse racionalmente como resultados de la investigación científica.

Desde la perspectiva de la TAD, la problemática didáctica es, esencialmente, una problemática económico-ecológica no normativa. Las problemáticas económica y ecológica están correlacionadas porque para estudiar las condiciones que permitirían y las restricciones que dificultan modificar el $\operatorname{PDV}_{\mathrm{I}}(\mathscr{D})$ en una dirección determinada, así como las consecuencias que acarrearía dicha modificación (su ecología), es imprescindible conocer su estructura y dinámica actuales (su economía). A su vez, para analizar la economía del $\mathrm{PDV}_{\mathrm{I}}(\mathfrak{D})$ es útil estudiar las restricciones que dificultan e incluso impiden su modificación. Además, para responder a las cuestiones relativas a la economía y la ecología del $\operatorname{PDV}_{\mathrm{I}}(\mathscr{D})$, se utiliza necesariamente la perspectiva que proporciona un $\operatorname{PDR}_{\mathrm{I}}(\mathscr{D})$ sustentado en un $\operatorname{MER}_{\mathrm{I}}(\mathfrak{D})$, lo que sitúa la problemática epistemológica en el núcleo de la problemática didáctica (Gascón, 2011).

\section{Construcción de un tipo particular de resultados de la investigación didáctica}

Supongamos que, al analizar la forma como se describen y utilizan los componentes praxeológicos (tipos de tareas, técnicas matemáticas y discursos tecnológico-teóricos) de cierto dominio $\mathscr{D}$ de las matemáticas escolares por parte de los sujetos de una institución escolar I, y observando los fines de la educación matemática que se persiguen en I con el estudio de $\mathscr{D}$, se detectan hechos notables, inicialmente sorprendentes, que admiten una descripción genérica y que se repiten regularmente en determinadas circunstancias y hasta en diferentes instituciones. Como consecuencia de ello, se considera que estos hechos requieren un estudio pormenorizado y para ello se empieza a construir, como hipótesis, un $\mathrm{PDR}_{\mathrm{I}}(\mathscr{D})$ lo que permitirá, simultáneamente, analizar más detalladamente y describir progresivamente una representación del $\operatorname{PDV}_{\mathrm{I}}(\mathscr{D})$.

Para contrastar empíricamente el $\operatorname{PDR}_{\mathrm{I}}(\mathscr{D})$ en construcción, se diseña un proceso de estudio del dominio $\mathscr{D}$ en la institución I protagonizado por una comunidad de estudio 
que cumple ciertas condiciones. Dicho proceso se sustenta en el $\operatorname{MER}_{\mathrm{I}}(\mathscr{D})$, y se lleva a cabo con los medios didácticos $\operatorname{MDR}_{\mathrm{I}}(\mathscr{D})$ que propone el $\operatorname{PDR}_{\mathrm{I}}(\mathscr{D})$ para perseguir los fines educativos $F_{R}(\mathscr{D})$ que este paradigma propugna y afrontar los fenómenos $\varphi_{R}(\mathscr{D})$. Se trata de diseñar un proceso de estudio regido por el paradigma $\operatorname{PDR}_{I}(\mathscr{D})$.

Presentamos a continuación dos ejemplos de leyes didácticas empíricamente sustentadas mediante procesos de estudio experimentales de este tipo. Cada uno de ellos proporciona respuestas (parciales y provisionales) a cuestiones que hemos citado anteriormente y que forman parte de la problemática económico-ecológica relativa a cierto $\operatorname{PDV}_{\mathrm{I}}(\mathscr{D})$. Los dos ejemplos se formulan con las herramientas que proporciona la TAD y, en cada caso, se utiliza un $\operatorname{PDR}_{\mathbf{I}}(\mathscr{D})$ subdisciplinar, construido como hipótesis por la investigación didáctica en cuestión, coherente con el paradigma didáctico disciplinar de la modelización matemática (PMM) (Gascón y Nicolás, 2021) para contrastarlo con el correspondiente $\operatorname{PDV}_{\mathrm{I}}(\mathscr{D})$. En cada ejemplo empezamos fijando la institución I y el dominio $\mathfrak{D}$ de las matemáticas que se toman en consideración y que desempeñan el papel de parámetros.

Ejemplo (1): $\mathrm{I}=\mathrm{S}=$ Secundaria; $\mathscr{D}=\mathscr{P}=$ Proporcionalidad

1.1. Si en la segunda etapa de la Enseñanza Secundaria Obligatoria española (14-16 años) se lleva a cabo el estudio de la proporcionalidad (suceso de tipo A), entonces dicho estudio estará regido por un $\operatorname{PDV}_{\mathrm{S}}(\mathscr{P})$ que comportará que la relación de proporcionalidad aparezca segregada del resto de relaciones funcionales y que se la identifique con una relación puramente aritmética entre números, lo que traerá consigo la evitación del uso del instrumento algebraico (suceso de tipo B).

1.2. Si en dicha institución, una comunidad — que cumpla ciertas condiciones a precisar - lleva a cabo un proceso de estudio de la proporcionalidad sustentado en un $\operatorname{MER}_{S}(\mathcal{P})$ del tipo presentado en García (2005), utilizando los mismos medios didácticos y persiguiendo los mismos fines educativos (suceso de tipo A), entonces dicha comunidad eludirá ${ }^{8} \operatorname{los}$ fenómenos didácticos habituales que aparecen cuando el estudio de la proporcionalidad está regido por el $\operatorname{PDV}_{\mathrm{S}}(\mathscr{P})$, empezando por la segregación de la relación de proporcionalidad. En coherencia con la nueva razón de ser que el $\operatorname{MER}_{\mathrm{S}}(\mathcal{P})$ asigna a la proporcionalidad, la comunidad de estudio evitará los fenómenos descritos en Bolea et al. (2001) y denominados, respectivamente, evitación del álgebra e identificación de la proporcionalidad con una relación puramente aritmética entre números (suceso de tipo B).

Ejemplo (2): I = SU = Tránsito de Secundaria a la Universidad; $\mathscr{D}=9 \mathfrak{F} \mathscr{F}=$ Modelización funcional

\footnotetext{
${ }^{8}$ Los fenómenos didácticos que estudiamos no son siempre “indeseables" desde la perspectiva que proporciona el paradigma didáctico que asumimos. Sin embargo, la decisión de estudiar un fenómeno - que, recordemos, es un hecho notable inicialmente asombroso que se repite regularmente en determinadas circunstancias - está motivada, en primer lugar, por la necesidad de explicarlo, lo que ya indica que un fenómeno siempre contrasta con lo que sería previsible desde la perspectiva de nuestros postulados. Pero, en segundo lugar, cuando decidimos estudiar un fenómeno didáctico lo hacemos, normalmente, por el deseo de actuar sobre el sistema en el que este aparece con la intención de modificarlo en algún sentido, por lo que tendemos a elegir fenómenos que, desde nuestra perspectiva, originan consecuencias "indeseables". Los criterios para elegir los fenómenos a estudiar y para decidir si son "indeseables" dependen de los postulados que asumimos y, en última instancia, se basan en un sistema de valores.
} 
2.1. Si en el tránsito entre la Enseñanza Secundaria y la Enseñanza Universitaria portuguesa (española o francesa) se lleva a cabo el estudio de la modelización funcional (suceso de tipo A), entonces dicho estudio estará regido por un $\mathrm{PDV}_{\mathrm{SU}}($ ๑) $\mathscr{F}$ ) que reducirá la actividad de modelización funcional al trabajo técnico dentro de unos pocos modelos funcionales dados de antemano. En este paradigma, el cálculo diferencial elemental desempeñará un papel accesorio y no se utilizará para construir modelos funcionales de diferentes tipos de sistemas, trabajar en ellos e interpretar los resultados obtenidos (suceso de tipo B).

2.2. Si en dicha institución, una comunidad - que cumpla ciertas condiciones a precisar - lleva a cabo un proceso de estudio de la modelización funcional sustentado en un MERsU(গ⿰冫广大 ) del tipo presentado en Lucas (2015), utilizando los mismos medios didácticos y persiguiendo los mismos fines educativos que se proponen en ese trabajo (suceso de tipo A), entonces dicha comunidad de estudio eludirá los fenómenos didácticos asociados a las limitaciones que aparecen cuando el estudio de la modelización

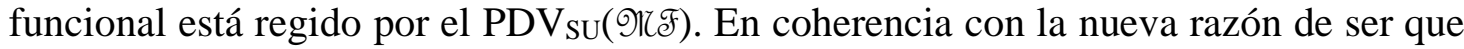

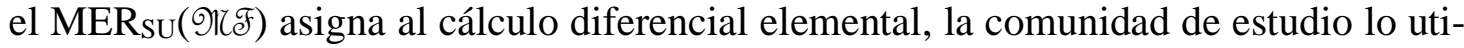
lizará, no sólo para estudiar las propiedades de los diferentes tipos de funciones, sino para construir modelos funcionales de sistemas de todo tipo, trabajar en ellos e interpretar los resultados obtenidos con el objetivo de responder cuestiones que surgen en dichos sistemas (suceso de tipo B).

Ambos ejemplos cumplen formalmente las condiciones para considerarse resultados que la ciencia didáctica puede formular legítimamente puesto que, según nuestra concepción de la investigación didáctica, esta puede guiarnos acerca de las acciones didácticas que podemos llevar a cabo (en determinadas condiciones) y de las consecuencias previsibles (queridas y no queridas) de dichas acciones, pero no puede proporcionarnos criterios acerca de las acciones didácticas que debemos realizar.

Los resultados ejemplificados son de dos tipos: o bien expresan las consecuencias de la vigencia de cierto paradigma didáctico en una institución determinada (visibles cuando este se analiza desde la perspectiva de un paradigma que se toma como referencia); o bien formulan enunciados sobre los medios racionalmente adecuados que permitirían conseguir fines previamente determinados, pero cuya validez no puede ser establecida racionalmente. En ningún caso prescriben ningún tipo de normas.

Al mismo tiempo, en ambos ejemplos salen a la luz fenómenos didácticos que requieren una explicación. Formulamos como sigue las preguntas cuya respuesta sería una explicación de dichos fenómenos en el sentido descrito en el apartado anterior:

1. ¿Por qué la proporcionalidad aparece en la institución de la enseñanza secundaria segregada del resto de relaciones funcionales elementales y es tratada como una relación puramente aritmética entre números (en vez de aparecer integrada como una relación funcional entre cantidades de magnitudes que requeriría el uso sistemático del instrumento algebraico)?

2. ¿Por qué, en el tránsito de la enseñanza secundaria a la enseñanza universitaria, el cálculo diferencial elemental desempeña, en el ámbito de la modelización funcional, un papel accesorio limitado al trabajo técnico para estudiar las características de unos pocos modelos funcionales dados de antemano (en lugar de utilizarse para construir modelos funcionales de sistemas de todo tipo, trabajar en ellos e interpretar los resultados obtenidos para responder a ciertas cuestiones)? 
Se trata de cuestiones relativamente abiertas, lo que significa que no tenemos una explicación ni completa ni definitiva de los fenómenos involucrados. Sin embargo, se han aportado respuestas parciales a ambas cuestiones respectivamente en García (2005), García et al (2007), Lucas (2015) y Lucas et al. (2017).

\section{5. ¿Para qué y cómo pueden utilizarse los resultados de la investigación?}

Hablamos de "utilizar" los resultados de la investigación en didáctica de las matemáticas, de diversas formas y con diferentes objetivos, en lugar de hablar de "aplicar" o "implementar" dichos resultados, porque ambos verbos, "aplicar" e "implementar", (quizá más el segundo que el primero) sugieren una relación muy directa entre resultados de la investigación y acción didáctica. No parece razonable suponer que los resultados obtenidos por una ciencia social, sea esta la didáctica u otra, puedan utilizarse tal cual, sin transformaciones previas, para actuar en el universo social.

Respecto a la cuestión de si es o no es posible utilizar legítimamente un resultado de la investigación para llevar a cabo acciones didácticas, queremos dejar claro, de entrada, que negar el carácter normativo o prescriptivo de la ciencia didáctica no implica en absoluto negar la posibilidad de utilizar legítimamente los resultados de la investigación en didáctica de las matemáticas para, entre otras cosas, incidir sobre determinados aspectos de la acción didáctica. La legitimidad dependerá, en última instancia, de las bases sobre las que se construyan dichos resultados y de la forma de utilizarlos que, por tanto, deberá ser especificada cuidadosamente.

Por esta razón, hemos ejemplificado un tipo particular (aunque potencialmente muy amplio) de problemas didácticos y el correspondiente tipo de resultados de investigación didáctica, construidos en base a los postulados metodológicos y epistemológicos de la TAD. Entre dichos postulados incluimos la asunción provisional, a modo de hipótesis, de una forma particular de interpretar la actividad matemática (un modelo epistemológico de las matemáticas), de unos fines de la educación matemática y, en definitiva, de un paradigma didáctico, el PMM (Gascón y Nicolás, 2021).

A continuación, formulamos, en términos generales, los dos tipos de resultados de la investigación didáctica involucrados en los ejemplos anteriores:

1. Si en una institución escolar I se lleva a cabo el estudio de cierto dominio $\mathscr{D}$ de las matemáticas, entonces dicho estudio estará regido por un paradigma, el $\operatorname{PDV}_{\mathrm{I}}(\mathscr{D})$, que se describe y se analiza desde la perspectiva de un $\operatorname{PDR}_{I}(\mathscr{D})$. El contraste entre ambos paradigmas sacará a la luz fenómenos didácticos que permanecían invisibles desde el punto de vista del $\operatorname{PDV}_{\mathrm{I}}(\mathfrak{D})$ y que habitualmente son considerados "indeseables" desde la perspectiva de un $\operatorname{PDR}_{I}(\mathscr{D})$.

2. Si en dicha institución se lleva a cabo un proceso de estudio del dominio $\mathfrak{D}$ regido por el paradigma $\operatorname{PDR}_{\mathrm{I}}(\mathfrak{D})$ construido para sacar a la luz los fenómenos didácticos citados, entonces a lo largo de dicho proceso de estudio se eludirán los fenómenos que emergen cuando el estudio de $\mathscr{D}$ está regido por el paradigma $\operatorname{PDV}_{\mathrm{I}}(\mathscr{D})$.

En lo que sigue nos centraremos en la cuestión del para qué y del cómo pueden utilizarse estos resultados. Para simplificar, consideraremos dos tipos de objetivos que pueden perseguirse con la utilización de los resultados de la investigación didáctica. Los podemos designar brevemente mediante dos etiquetas: aumentar los conocimientos sobre el universo didáctico e incidir sobre la investigación y la acción didácticas. 
En cualquier caso, no debemos olvidar que, en el estado actual de la ciencia didáctica, la mayoría de los resultados de la investigación en didáctica de las matemáticas, incluyendo los que hemos ejemplificado, son todavía provisionales, están poco contrastados empíricamente, y se refieren a un dominio particular relativamente reducido de las matemáticas, a una institución concreta y, en muchos casos, su base empírica se reduce a unas pocas experimentaciones, por lo que su utilización a gran escala se vuelve problemática y debe matizarse y formularse con mucha prudencia.

\subsection{Aumentar los conocimientos sobre el universo didáctico}

Los resultados de la investigación didáctica descritos constituyen respuestas parciales a cuestiones o problemas didácticos que habíamos planteado anteriormente. Dichos resultados proporcionan características de la economía y de la ecología del PDV $\operatorname{PD}_{\mathrm{I}}(\mathfrak{D})$ de dos dominios de las matemáticas escolares: la proporcionalidad en la enseñanza secundaria obligatoria y el cálculo diferencial elemental en el ámbito de la modelización funcional, en el tránsito de la Secundaria a la Universidad. En cada uno de dichos ejemplos se describen fenómenos didácticos que permanecían invisibles desde el punto de vista institucional y se propone una forma de superar ciertas "limitaciones" que muestra la educación matemática vigente en I en torno a $\mathscr{D}$, cuando esta se observa desde la perspectiva que proporciona cierto $\operatorname{PDR}_{I}(\mathscr{D})$.

En consecuencia, el primer objetivo que se obtiene a partir de los resultados de la investigación didáctica, como sucede con los resultados obtenidos en cualquier otra actividad científica, consiste en aumentar nuestros conocimientos sobre una parte del mundo. Con más precisión, estos resultados de la investigación en didáctica de las matemáticas sirven, en primer lugar, para aumentar los conocimientos sobre los paradigmas didácticos vigentes en una institución (en torno a un cierto dominio de las matemáticas) y, en particular, para sacar a la luz y empezar a dar cuenta de determinados fenómenos didácticos que emergen en los procesos de estudio de dicho dominio.

\subsection{Incidir sobre la investigación y la acción didácticas}

Nos preguntamos ahora cómo se puede utilizar un resultado de la investigación didáctica para incidir localmente sobre la acción didáctica y la investigación. Empezamos por las acciones didácticas involucradas en el diseño, la gestión y la evaluación de un proceso de estudio en torno a un dominio $\mathscr{D}$ de la matemática escolar relativamente acotado como la divisibilidad, la proporcionalidad, la trigonometría, la geometría analítica del plano, el cálculo diferencial elemental o la estadística inferencial.

Pueden plantearse acciones didácticas más ambiciosas, que abarquen un ámbito más amplio del universo didáctico, como la construcción de un currículo completo para la enseñanza de las matemáticas en determinados niveles educativos o la elaboración de un programa para la formación del profesorado. Los resultados de la investigación didáctica que se pretende utilizar no tienen que referirse necesariamente, como sucede en nuestros dos ejemplos, a un dominio matemático particular más o menos amplio. Podría tratarse de resultados de la investigación didáctica relativos a la enseñanza de la resolución de problemas o de la demostración matemática o, incluso, a la forma de organizar los procesos de estudio de las matemáticas. Sin embargo, estas propuestas globales o transversales estarán siempre mejor fundamentadas científicamente si están basadas en la articulación de resultados obtenidos en investigaciones didácticas locales coherentes 
entre sî́ $^{9}$. En todo caso, aquí nos ceñimos a los tipos de resultados de la investigación de los que forman parte los ejemplos descritos y que están referidos a un dominio relativamente acotado de la matemática escolar.

En primer lugar, cada uno de estos resultados puede guiar el diseño, la gestión y la evaluación de procesos de estudio regidos por el nuevo $\operatorname{PDR}_{\mathrm{I}}(\mathfrak{D})$ con el objetivo de replicar, precisar y consolidar el resultado en cuestión. Para ello será necesario ir concretando progresivamente, mediante la explicitación de indicadores empíricos, las condiciones iniciales que debe satisfacer la comunidad de estudio y las características precisas que debe presentar el $\operatorname{PDV}_{\mathrm{I}}(\mathscr{D})$ para que dicha comunidad esté en condiciones de vivir un proceso de estudio regido por el $\operatorname{PDR}_{\mathrm{I}}(\mathfrak{D})$. También deberán precisarse los indicadores que permitan evaluar en qué medida la puesta en marcha de los medios didácticos $\operatorname{MDR}_{\mathrm{I}}(\mathscr{D})$ que propone el $\operatorname{PDR}_{\mathrm{I}}(\mathfrak{D})$ permiten que la comunidad de estudio alcance los fines $F_{R}$ de la educación matemática y que salgan efectivamente a la luz los fenómenos $\varphi_{R}$. Las sucesivas experimentaciones de procesos de estudio regidos por el $\operatorname{PDR}_{\mathrm{I}}(\mathscr{D})$ permitirán consolidar y, en su caso, generalizar el resultado correspondiente y desarrollar así la investigación didáctica.

En segundo lugar, una vez precisado y (relativamente) consolidado un resultado de este tipo, tendremos el contraste entre un $\operatorname{PDR}_{\mathrm{I}}(\mathscr{D})$, construido por la TAD y compatible con el PMM, y el $\mathrm{PDV}_{\mathrm{I}}(\mathscr{D})$. Este resultado no nos dirá cómo se debe conceptualizar el dominio $\mathscr{D}$, cuál es el modelo epistemológico más adecuado para representarlo, qué medios didácticos deben ponerse en marcha para organizar su estudio en I, ni qué argumentos deben utilizarse a lo largo del proceso de estudio. Tampoco nos dirá cuáles son los fines educativos que deben perseguirse con dicho estudio o los fenómenos que se deben evitar o favorecer. El resultado sí afirmará que existe una conceptualización del dominio $\mathscr{D}$, representada por el $\operatorname{MER}_{\mathrm{I}}(\mathscr{D})$ y unos medios didácticos $\operatorname{MDR}_{\mathrm{I}}(\mathscr{D})$ que son útiles y racionalmente adecuados para que la comunidad de estudio alcance los fines $\mathrm{F}_{\mathrm{R}}(\mathfrak{D})$ mediante un proceso de estudio en el que utilizarán argumentos racionales (no necesariamente deductivos), y se evitarán (o favorecerán) fenómenos didácticos invisibles desde la perspectiva del $\operatorname{PDV}_{\mathrm{I}}(\mathscr{D})$. Dado que el $\mathrm{PDR}_{\mathrm{I}}(\mathscr{D})$ es un PD subdisciplinar coherente con el PMM, la fundamentación racional de la verdad de los conocimientos se basará, en parte, en las relaciones entre propiedades del sistema a modelizar, definido en términos de magnitudes en los ejemplos anteriores, y propiedades del modelo matemático de dicho sistema (Bochaca et al., 2021).

En este punto se pone de manifiesto que la posible utilización de los resultados de la investigación en el ámbito de la acción didáctica no es ni directa ni inmediata. En efecto, para que sea posible empezar a utilizar este resultado en la acción didáctica, se requiere, en primer lugar, que la comunidad educativa decida (en base a un determinado sistema de valores) que los fines de referencia $F_{R}$ son más valiosos que los fines vigentes $\mathrm{F}_{\mathrm{V}}$ y que ciertos fenómenos didácticos deben ser evitados (o favorecidos). Se requiere, asimismo, que dicha comunidad acepte (y sea capaz de) poner en marcha unos medios didácticos $\operatorname{MDR}_{\mathrm{I}}(\mathscr{D})$ que pueden ser muy diferentes a los medios vigentes $\operatorname{MDV}_{\mathrm{I}}(\mathscr{D})$ habituales. Estos $\operatorname{MDR}_{\mathrm{I}}(\mathscr{D})$ pueden organizarse en términos de recorridos de estudio e

\footnotetext{
${ }^{9}$ Un ejemplo de propuesta global no sustentada en investigaciones didácticas locales lo constituye el documento Bases para la elaboración de un currículo de Matemáticas en Educación no Universitaria publicado por el Comité Español de Matemáticas (CEMat) en mayo de 2021.
} 
investigación (REI) en la línea del "inquiry based mathematics education" (García et al., 2019, pp. 80-82) que son dispositivos didácticos especialmente adecuados para superar las restricciones que dificultan la vida escolar de la modelización matemática (Barquero et al., 2011), o bien en términos de juegos didácticos de indagación (Bochaca et al., 2021). Pero, en todos los casos, lo que es más problemático, si se pretende utilizar este resultado en la acción didáctica a gran escala (más allá de las experimentaciones locales), es controlar el impacto que tendrán, sobre la educación matemática en I, las profundas transformaciones que sufrirá la educación matemática en torno a $\mathfrak{D}$. Nada nos asegura que dichas transformaciones (la nueva forma de conceptualizar $\mathscr{D}$ y sus nuevas relaciones con el resto de los dominios de la matemática escolar, los nuevos fines educativos asociados al estudio de $\mathfrak{D}$ en I, los nuevos medios didácticos, etc.) sean ecológicamente compatibles con la educación matemática en I globalmente considerada.

¿En qué consiste, por tanto, la utilidad de los resultados de la investigación en lo que respecta a su incidencia sobre la acción didáctica? En general, podemos decir que, como en todas las ciencias, lo que puede ser útil para modificar la acción (didáctica) se desprende de las hipótesis atrevidas y fecundas que propone la ciencia (didáctica). Por ejemplo, sería interesante preguntarse cuál ha sido y sigue siendo la utilidad de los resultados de la ciencia económica o de la física nuclear en la acción social. Dichos resultados, ¿prescriben normas para la acción o proporcionan posibilidades de acción? En el ámbito de la TAD, y restringiéndonos al tipo de problemas didácticos presentados, los resultados de la investigación se formulan mediante hipótesis que se materializan en un $\operatorname{PDR}_{\mathrm{I}}(\mathscr{D})$. Su utilidad consiste en ofrecer posibilidades de intervenciones (relativamente) realizables y normalmente locales sobre la acción didáctica.

Pensando en el desarrollo futuro de la ciencia didáctica, dado que la TAD asume a nivel disciplinar el PMM y que todos $\operatorname{los} \operatorname{PDR}_{\mathrm{I}}(\mathscr{D})$ subdisciplinares que construye son compatibles con dicho paradigma (Gascón y Nicolás, 2021, pp. 30-33), se abre la posibilidad de articularlos para diseñar un paradigma didáctico disciplinar. Este paradigma podría ser compartido, en particular, en la institución de la enseñanza secundaria de las matemáticas. Dicha construcción requerirá un desarrollo de la investigación didáctica y, potencialmente, proporcionaría la posibilidad de llevar a cabo una acción didáctica global — con la participación de equipos mixtos de profesores e investigadores - basada en la articulación de múltiples investigaciones locales.

\section{Agradecimientos:}

Esta investigación se ha financiado por los proyectos I+D+i: RTI2018-101153-AC22 (MCIU/AEI/FEDER, UE) y RTI2018-101153-B-C21 (MCIU/AEI/FEDER, UE)

\section{Referencias}

Barquero, B., Bosch, M. y Gascón, J. (2011). Los Recorridos de Estudio e Investigación y la modelización matemática en la enseñanza universitaria de las ciencias experimentales. Enseñanza de las Ciencias, 29(3), 339-352.

Bartolini Bussi, M. G. (2018). Answer to Gascón \& Nicolás. For the Learning of Mathematics, 38(3), 50-53.

Bochaca, P., Gascón, J. y Nicolás, P. (2021). Juegos didácticos de indagación en torno a la covariación de dos magnitudes continuas. Caminhos da Educacão Matemática, 11(1), 223-250. 
Bolea, P., Bosch, M. y Gascón, J. (2001). La transposición didáctica de organizaciones matemáticas en proceso de algebrización. El caso de la proporcionalidad. $R e$ cherches en Didactique des Mathématiques, 21(3), 247-304.

Bourdieu, P. (2001). Science de la science et réflexivité. Editions Raisons d'Agir.

Chevallard, Y. (2011). Quel programme pour l'avenir de la recherche en TAD ? En M. Bosch, J. Gascón, A. Ruiz Olarría, M. Artaud, A. Bronner, Y. Chevallard, G. Cirade, C. Ladage y M. Larguier (eds.), Un panorama de la TAD (pp. 23-32). CRM Documents, vol. 10. Centre de Recerca Matemàtica.

Chevallard, Y., Barquero, B., Bosch, M., Florensa, I., Gascón, J., Nicolás, P. y RuizMunzón, N. (en prensa). Advances in the Anthropological Theory of the Didactic. Birkhauser-Springer.

Davis, B. (2018). What sort of science is didactics? For the Learning of Mathematics, $38(3), 44-49$.

García, F. J. (2005). La modelización como herramienta de articulación de la matemática escolar. De la proporcionalidad a las relaciones funcionales. Trabajo de Tesis Doctoral sin publicar. Universidad de Jaén.

García, F. J., Barquero, B., Florensa, I. y Bosch, M. (2019). Diseño de tareas en el marco de la Teoría Antropológica de lo Didáctico. Avances de Investigación en Educación Matemática, 15, 75-94. https://doi.org/10.35763/aiem.v0i15.267

García, F. J., Bosch, M., Gascón, J. y Ruiz Higueras, L. (2007). Integración de la proporcionalidad escolar en una organización matemática regional en torno a la modelización funcional: Los planes de ahorro. En L. Ruiz-Higueras, A. Estepa y F. J. García (eds.), Sociedad, Escuela y Matemáticas. Aportaciones de la Teoría Antropológica de lo Didáctico (pp. 439-460). Universidad de Jaén.

García, R. (2011). Interdisciplinariedad y sistemas complejos. Revista Latinoamericana de Metodología de las Ciencias Sociales, 1(1), 66-100.

Gascón, J. (1997). The Didactics of Mathematics as the Science of the "Art of Studying", En N. A. Malara (ed.), An International View on Didactics of Mathematics as a Scientific Discipline (ICME8, pp. 7-12). University of Modena.

Gascón, J. (2001). Incidencia del modelo epistemológico de las matemáticas sobre las prácticas docentes. RELIME, 4(2), 129-159.

Gascón, J. (2011). Las tres dimensiones fundamentales de un problema didáctico. El caso del álgebra elemental. RELIME, 14(2), 203-231.

Gascón, J. (2021). ¿Qué tipo de resultados puede enunciar legítimamente la ciencia didáctica? Simposio Latinoamericano de Didática da Matemática (LADIMA), (online).

Gascón, J. y Nicolás, P. (2017). Can didactics say how to teach? The beginning of a dialogue between the anthropological theory of the didactic and other approaches. For the Learning of Mathematics, 37(3), 26-30.

Gascón, J. y Nicolás, P. (2019a). Economía, ecología y normatividad en la teoría antropológica de lo didáctico. Educação Matemática Pesquissa, 21(4), 36-52. (Versión original 2016). https://doi.org/10.23925/1983-3156.2019v21i4p036-052

Gascón, J. y Nicolás, P. (2019b). Research ends and teaching ends in the anthropological theory of the didactic. For the Learning of Mathematics, 39(2), 42-47. 
Gascón, J. y Nicolás, P. (2019c). What kind of results can be rationally justified in didactics? En M. Bosch, Y. Chevallard, F. J. García y J. Monaghan (eds.), Working with the anthropological theory of the didactic in mathematics education: A comprehensive casebook (pp. 3-11). Routledge. https://doi.org/10.4324/9780429198168-1

Gascón, J. y Nicolás, P. (2021). Incidencia de los paradigmas didácticos sobre la investigación didáctica y la práctica docente. Educación Matemática, 33(1), 7-40.

Gascón, J. y Nicolás, P. (en prensa). Crisis of paradigm in the step from tertiary to secondary mathematics education. International Journal of Mathematical Education in Science.

Godino, J. D., Batanero, C. y Font, V. (2019). The onto-semiotic approach: Implications for the prescriptive character of didactics. For the Learning of Mathematics, 39(1), 38-43.

Lerman, S. (2018). Towards subjective truths in mathematics education. For the Learning of Mathematics, 38(3), 54-56.

Lucas, C. (2015). Una posible razón de ser del cálculo diferencial elemental en el ámbito de la modelización funcional. Trabajo de Tesis Doctoral sin publicar. Universidad de Vigo.

Lucas, C., Gascón, J. y Fonseca, C. (2017). Razón de ser del Cálculo Diferencial Elemental en la transición entre la enseñanza secundaria y la universitaria. REDIMAT, 6(3), 283-306. https://doi.org/10.17583/redimat.2017.2116

Mosterín, J. y Torretti, R. (2010). Diccionario de Lógica y Filosofía de la Ciencia. Alianza Editorial.

Oktaç, A., Trigueros, M. y Romo, A. (2019). APOS Theory: Connecting research and teaching. For the Learning of Mathematics, 39(1), 33-37.

Proulx, J. (2018). Prescriptions and proscriptions on mathematics teaching: interesting cases of lost in translation. For the Learning of Mathematics, 38(3), 56-57.

Staats, S. y Laster, L. A. (2019). About time. For the Learning of Mathematics, 39(1), 44-47.

Weber, M. (2009). La "objetividad" del conocimiento en la ciencia social y en la política social. Alianza Editorial. (Versión original 1904).

Weber, M. (2010). Por qué no se deben hacer juicios de valor en la sociología y en la economía. Alianza Editorial. (Versión original 1917).

\section{Referencias de los autores}

Josep Gascón, Universitat Autònoma de Barcelona (España), gascon@mat.uab.cat

Pedro Nicolás, Universidad de Murcia (España), pedronz@um.es 


\title{
Relationship between teaching and research in didactics of mathematics
}

\author{
Josep Gascón, Universitat Autònoma de Barcelona \\ Pedro Nicolás, Universidad de Murcia
}

Taking Weber, science speaks of facts and does not produce, as research results, normative prescriptions and value judgments. However, those prescriptions and judgments have a strong presence in academic literature in didactics. We thus find of interest and even urgent to address the many questions around the relationship between teaching and research in didactics. In this article we explain our point of view within the framework of the anthropological theory of the didactic. Let us assume that, in order to analyse the way a certain domain $\mathscr{D}$ of mathematics is studied in an educative institution $I$ (i.e. which are the task types, which are the techniques studied to face those types, which is the technological-theoretical discourse, how the institution $I$ justifies the study of $\mathscr{D}$ ), one notices a remarkable surprising fact, $e$. Let us imagine this fact admits a generic description and persists regularly, as a characteristic of the institution $I$ itself. If $e$ is surprising is because one could imagine an alternative fact $d$ instead. Then it makes sense to ask why does the event e occur (rather than the event $d, \ldots$...)? When trying to answer this question one naturally finds some other related issues. A serious attempt to provide an answer to all those interrogations requires a description of the event $e$ and of the alternative event $d$. The description of $e$ gives rise to a type of didactic laws, general statements describing a state of things concerning the study of $\mathscr{D}$ in the institution $I$ which takes place in a certain constant way. There is another type of didactic laws, those counterfactual which state that a certain series of actions would allow the occurrence of the event $d$ instead of $e$. To address and formulate all these questions, we propose the notion of didactic paradigm, essentially made of the educational ends attached to the study of $\mathscr{D}$ in $I$ (the answer to why should $\mathscr{D}$ be studied in $I$ ?) and the epistemological model for $\mathscr{D}$ in $I$ (the answer to how is the learning of $\mathscr{D}$ conceptualized in $I$ ?). We use two different kinds of didactic paradigms, distinguished in what they represent. On the one hand and to describe $e$, we use the current didactic paradigm, representing the way in which $\mathscr{D}$ is studied in $I$. On the other hand and to describe $d$, we use the so-called reference didactic paradigm, representing an alternative way of studying $\mathscr{D}$ in $I$. Whereas in the choice of the surprising fact $e$ and the alternative $d$ certain values unavoidably take part, those values do not appear in the statements of didactic laws. 